\title{
Non-coaxial plastic flow of granular materials through stress probing analysis
}

\author{
Mehdi Pouragha ${ }^{\mathrm{a}, *}$, Niels P. Kruyt ${ }^{\mathrm{b}}$, Richard Wan ${ }^{\mathrm{c}}$ \\ ${ }^{a}$ Civil and Environmental Engineering Department, Carleton University, Canada \\ ${ }^{\mathrm{b}}$ Department of Mechanical Engineering, University of Twente, The Netherlands

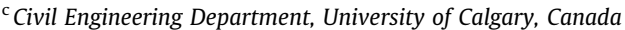

\section{A R T I C L E I N F O}

\section{Article history:}

Received 24 August 2020

Received in revised form 25 January 2021

Accepted 1 March 2021

Available online 11 March 2021

\section{Keywords:}

Granular materials

Plasticity

Non-coaxiality

Multi-mechanism plasticity

Discrete element method simulation

Stress probes

\begin{abstract}
A B S T R A C T
The current study investigates the non-coaxial plastic flow in granular media where the principal directions of incremental plastic strain differ from those of the current stress (and of the current fabric tensor). The stress probing technique is used with Discrete Element Method simulations to capture the plastic response of a 2D granular assembly to loading increments along different directions in stress space, including non-coaxial ones. A general form for the plastic flow is suggested, based on multimechanism plasticity theory which is demonstrated to be capable of capturing the essential characteristics of the non-coaxial plastic flow. Deviations between DEM results and model predictions are associated with the incrementally nonlinear nature of the response. The analysis of the results indicate that, at a given stress state, the plastic yielding direction is not coaxial with the current stress. The significance of the non-coaxial plastic flow is shown to be around $20 \%$ for lower deviatoric stresses, but this value diminishes as the peak stress is achieved, hinting to the dominance of a single mechanism at failure. The study concludes by comparing the developed model with two well-known non-coaxial models in the literature, where the performance of the developed model is found to be close to the doubleshearing theory.
\end{abstract}

(c) 2021 Elsevier Ltd. All rights reserved.

\section{Introduction}

The classical theory of plasticity formalized by Hill (1998) forms the basis of most constitutive models for the inelastic deformation of geomaterials, including heterogeneous media such as granular materials. Based on smooth yield surfaces and plastic potentials, plastic flow is described through a relationship between plastic strain and stress increments (or rates) following some postulates. Firstly, according to Ziegler's orthogonality postulate (Ziegler, 1970; Houlsby and Puzrin, 2007), plastic strains are deemed to be independent of stress increment direction so that they plot as a unique line in strain rate space (corresponding to the gradient of the plastic potential). Secondly, it is postulated that the principal directions of the plastic strain increment coincide with those of the current stress tensor, the so-called coaxiality condition.

However, it is well recognized that the above two idealizations break down in the case of granular materials, especially under complex loading, as discussed in Kuhn and Daouadji (2018). The ensuing impacts on granular materials are indeed significant as

\footnotetext{
* Corresponding author.

E-mail address: mehdi.pouragha@carleton.ca (M. Pouragha).
}

they permeate into prominent attributes such as dilatancy, plastic dissipation and strain localization properties (Anand, 1983; Loret, 1992; Lade et al., 2009; Kuhn and Daouadji, 2018; Gutierrez et al., 1991; Gutierrez and Ishihara, 2000). Among others, such deviations from classical plasticity are often studied under the formalism of "incremental non-linearity" and "non-coaxiality". Here, the former refers to the dependence of the material response on loading direction beyond the simple loading/unloading dichotomy involving two tensorial zones considered within incrementally linear plasticity frameworks (Darve, 1990; Darve and Nicot, 2005).

The non-coaxiality phenomenon refers to the deviation between principal directions of stress tensor and plastic strain rate, which is the main focus of the current study. The experimental manifestation of non-coaxiality dates back to the early work of De Josselin de Jong (1959), Drescher and de Josselin de Jong (1972) on analogue granular materials consisting of photoelastic rods. Subsequently, there have been numerous demonstrations of non-coaxiality in hollow cylinder tests by, among others, Gutierrez et al. (1991), Yoshimine et al. (1998) and Yang et al. (2007) where the directions of principal stresses can be controlled while exploring the strain response. An overview of both experimental and micromechanical evidences for the importance of 
non-coaxiality on the behaviour of granular materials is given in Yu (2006), where various non-coaxial constitutive models are also described.

From a theoretical perspective, there have been several attempts over the years to incorporate the effect of non-coaxial loading into the theory of plasticity, which, based on their point of departure, fall into two broad groups.

The first group includes studies originating from the double shearing theory based on pioneering ideas of Spencer (1964) and further developed in Mehrabadi and Cowin (1978), which are able to capture, albeit indirectly, the non-coaxial loading effects on plastic flow. Unlike the conventional elasto-plastic formulation which is based upon a unique plastic flow direction and dilatancy value, the double-shearing theory assumes a constant ratio between normal and shear strains along the mobilized slip-lines, leading to a dilatancy value which varies according to loading direction. Modern continuations of the double-shearing theory and its application to non-coaxiality can be found in more recent works (Harris, 1995; Nemat-Nasser and Zhang, 2002; Zhu et al., 2007; Yu and Yuan, 2006).

The second group of theories has been developed by Tsutsumi and Hashiguchi (2005), Tsutsumi et al. (2005), whereby the classic elasto-plastic theory is extended by explicitly incorporating extra terms in the plastic flow equation that capture the non-coaxial loading effects. Both double shearing and Hashiguchi theories, capable as they are in incorporating non-coaxiality, still fall under the category of incrementally linear models.

Li and Dafalias (2004) also incorporated the effect of noncoaxiality into their series of constitutive models by directly assuming an additive decomposition of plastic flow into the contributions originating from monotonic loading and stress rotation. More recent works on models based on bounding surface plasticity and the concept of back-stress have also proven to be capable of capturing the non-coaxial responses by assuming dependency of flow rules and bounding surfaces on the loading direction (Dafalias and Taiebat, 2016). The eventual formulation resembles the interpretation of double-shearing models (Nemat-Nasser and Zhang, 2002; Yu and Yuan, 2006) which equate to having separate terms for the plastic flow attributed to stress rotation.

As another notable class, extensions to constitutive models with combined isotropic/kinematic hardening plasticity have also been made to describe how the principal directions of strain rate evolve with those of stress, see e.g. de Boer and Brauns (1990), Papamichos and Vardoulakis (1995), Qian et al. (2013).

On the other hand, micromechanical origins of non-coaxial plastic responses have been traced down to particle-scale kinematics and micro-slips at contact points, as well as the topology of the contact network and grain rearrangement, see for instance Zhu et al. (2007), Li and Yu (2015), Gao and Zhao (2017), Shi et al. (2020), Balendran and Nemat-Nasser (1993). In particular, the non-coaxiality between stress and plastic strain increments is often correlated with the non-coaxiality between current contact fabric and its incremental evolution, contact fabric and strain rates, or interparticle contact and force distributions ( $\mathrm{Li}$ and Yu, 2015; Gao and Zhao, 2017; Li and Yu, 2014).

The two concepts of incremental non-linearity and noncoaxiality are different in that the latter describes the plastic strain rate dependence on the rotation of principal stress directions, while the former describes higher orders of dependency of material response on loading direction. As such, the two can theoretically exist independently of each other. On the other hand, these concepts are correlated in that they both describe more elaborate dependencies of plastic strain rate on loading direction.

Such directional dependencies on loading direction are best studied via stress (or strain) probing in which increments of stress (strain) are applied in different directions, but with con- stant magnitude, and resulting strain (stress) increments are recorded and analyzed. This technique was originally proposed by Gudehus (1979) to study the characteristic nature of constitutive models.

Although the probing technique is difficult to perform experimentally (Hareb and Doanh, 2012), it is most amenable to numerical simulations such as Finite Element or Discrete Element Method (DEM) (Cundall and Strack, 1979) where the same numerically prepared sample can be repeatedly probed along different loading directions. For instance, using DEM simulations of stress probes, incrementally nonlinear behaviour has been investigated by Bardet (1994), Calvetti et al. (2003), Froiio and Roux (2009), Wan and Pinheiro (2014), Darve and Nicot (2005), Darve and Nicot (2005). However, these studies have focused mostly on incremental loading programmes that do not involve stress rotation, thus not investigating possible non-coaxiality effects as generally as in non-proportional loading.

To extend upon these previous studies, we herein use DEM simulations to study an extensive sets of probes including loading directions that are non-coaxial with respect to the current stress. To avoid the complexity in interpretation and to limit the number of DEM simulations, the two-dimensional case is considered here. Furthermore, we limit our study to cases where the initial stress and fabric tensors are coaxial and as such, there is no distinction between the non-coaxiality with respect to these tensors. A straightforward decomposition of plastic flow into coaxial and noncoaxial parts is used for the 2D case considered whereas a general 3D decomposition is described by Li and Dafalias (2020). A double-mechanism plasticity formulation is adopted where the non-coaxial effects are naturally captured by a second set of plastic flow directions. For the considered 2D cases, the plastic flow formulation is shown to attain simplified mathematical forms that resemble those in Tsutsumi and Hashiguchi (2005), but with subtle differences that will be highlighted later in this paper.

The plastic flow response obtained from DEM simulations exhibits systematic deviations from coaxial flow theory, hence noncoaxiality, as well as incremental non-linearity. The proposed formulation is shown to be capable of capturing the non-coaxial phenomenon within reasonable accuracy, while suggestions are provided for incorporating incremental non-linearity. As an essential finding, the numerical results indicate that, contrary to the common assumption in elasto-plasticity, the yielding direction in stress space is generally not coaxial with the current stress despite the fabric tensor being coaxial here. Interestingly, the multimechanism behaviour (leading to non-coaxiality) is found to be more prevalent in the initial stages of deviatoric loading, and as the peak stress is approached, a single dominant mechanism emerges to govern the plastic flow response. Finally, for completeness, the model performance is compared with Hashiguchi's formulation (Tsutsumi and Hashiguchi, 2005) and double-shearing theory (Mehrabadi and Cowin, 1978) both qualitatively and quantitatively.

The outline of this study is as follows. Section 2 describes the non-coaxial plastic flow formulation in the context of multimechanism plasticity. In Section 3, the DEM simulations of the stress probing procedure are detailed out. The results of the probe simulations are analyzed in Section 4. The findings of this study are discussed in Section 5, with final conclusions and recommendations given in Section 6 .

\subsection{Notation conventions}

The following notation is used in this study. For an arbitrary tensor $\mathbf{A}$, the norm $|\mathbf{A}|$ and the corresponding normalized "direction" tensor $\hat{\mathbf{A}}$ are defined by 


$$
|\mathbf{A}|=\sqrt{\mathbf{A}: \mathbf{A}}=\sqrt{A_{i j} A_{j i}} \quad \hat{\mathbf{A}}=\frac{\mathbf{A}}{|\mathbf{A}|} .
$$

The rate of change with time of a tensor $\mathbf{A}$ is denoted by $\dot{\mathbf{A}}$. No distinction is made between $\dot{\mathbf{A}}$ and objective rates (such as the Jaumann rate), since only small strains are considered here. The Macauley bracket $\langle x\rangle$ of a scalar quantity $x$ equals zero if $x<0$ and $x$ if $x \geqslant 0$.

In the two-dimensional case considered here, the mean pressure $p$, the deviatoric stress tensor $\tau$ and the (scalar) stress deviator $q$ are defined by

$p=\frac{1}{2} \operatorname{tr}(\boldsymbol{\sigma}) \quad \tau=\boldsymbol{\sigma}-p \boldsymbol{\delta} \quad q=\sqrt{\frac{1}{2} \tau: \tau}$,

where $\delta$ is the identity tensor.

Moreover, the vector representation of a symmetric, secondorder tensor $\mathbf{A}$ is given by

$$
\left[\begin{array}{ll}
A_{11} & A_{12} \\
A_{12} & A_{22}
\end{array}\right] \rightarrow\left[\begin{array}{c}
A_{11} \\
A_{22} \\
\sqrt{2} A_{12}
\end{array}\right]
$$

The coefficient $\sqrt{2}$ is included in order that the norm of the tensor and the vector representation be equal.

\section{Plastic flow formulation}

Assuming an additive decomposition of infinitesimal strain rate into elastic and plastic parts, an incremental relation can be written between plastic strain and stress rates in the following generic form

$\dot{\boldsymbol{\varepsilon}}^{p}=\mathbb{C}(\boldsymbol{\sigma}, \hat{\boldsymbol{\sigma}}): \dot{\boldsymbol{\sigma}}$

where $\dot{\boldsymbol{\varepsilon}}^{p}$ and $\dot{\boldsymbol{\sigma}}$ are the rates of the plastic strain and the stress tensors respectively, $\mathbb{C}$ is the fourth-order plastic compliance tensor, and $\hat{\dot{\boldsymbol{\sigma}}}=\dot{\boldsymbol{\sigma}} /|\dot{\boldsymbol{\sigma}}|$ is the direction of the stress increment (loading direction), defined through Eq. 1. Possible dependency of the plastic flow on the loading direction $\hat{\boldsymbol{\sigma}}$ leads to the class of incrementally nonlinear models (Darve, 1990).

The flow nature of plastic deformation requires the plastic operator $\mathbb{C}$ to be rank deficient with at least one zero eigenvalue (Itskov, 2000). Therefore, the plastic strain response, $\dot{\boldsymbol{\varepsilon}}^{p}$, resides in a subspace with dimension of $D-1$ or lower, with $D$ being the number of independent elements in the rate of stress and plastic strain tensors. A similar conclusion has been obtained through micromechanical analyses in Goddard and Bashir (1990), Bashir and Goddard (1991), Kruyt (1993), Kruyt and Rothenburg (2004) where the analysis of the dilatancy behaviour of relatively stiff granular materials leads to a geometrical constraint between the contact fabric (Horne, 1965) and the strain rate tensors.

We denote here by "conventional" the elasto-plastic frameworks that are based on a single set of yield surface and plastic potential that depends only on the current stress state. The conventional plastic flow rule is therefore recovered for simple cases with $D=2$ (such as in triaxial loading), where the fourth-order rank-deficient plastic compliance tensor $\mathbb{C}$ can be immediately expressed as the dyadic product of two normalized second-order tensors $\hat{\mathbf{N}}$ and $\hat{\mathbf{M}}$, i.e., $C_{i j k l}=\Lambda \widehat{N}_{i j} \widehat{M}_{k l}$ :

$\dot{\boldsymbol{\varepsilon}}^{p}=\Lambda\langle\hat{\mathbf{M}}: \dot{\boldsymbol{\sigma}}\rangle \hat{\mathbf{N}}$

Here the Macauley brackets $\langle$.$\rangle distinguish between loading and$ unloading tensorial zones, with plastic strain only occurring within the loading zone. The form of Eq. 5 is analogous to the flow rule in conventional plasticity (Pastor et al., 1990) with $\hat{\mathbf{M}}$ and $\hat{\mathbf{N}}$ representing the gradient of the yield surface and the plastic potential functions, respectively.

The conventional single mechanism plastic flow depicted by Eq. 5 is based upon a number of simplifying assumptions, many of which break down under more elaborate loadings, as elegantly documented and analyzed by Kuhn and Daouadji (2018). As mentioned earlier, the transition from Eq. 4 to 5 is not guaranteed for cases with more than two degrees of freedom. Instead, for such cases, the plastic response has been reported to reside in a hyperplane (Kuhn and Daouadji, 2018; Nicot and Darve, 2007; Wan and Pinheiro, 2014; Harthong and Wan, 2013). In these cases, Eq. 5 is recovered only by explicitly adopting Ziegler's orthogonality condition (Houlsby and Puzrin, 2007) which predisposes the compliance tensor $\mathbb{C}$ with $D-1$ zero eigenvalues.

\subsection{Multi-mechanism plasticity}

If Ziegler's orthogonality condition is relaxed, a multimechanism formulation can be adopted whereby $\mathbb{C}$ within the plastic (loading) tensorial zone is given by:

$\mathbb{C}=\sum_{i=1}^{D-1} \Lambda^{i} \hat{\mathbf{N}}^{i} \hat{\mathbf{M}}^{i}$

with $\Lambda^{1}$ to $\Lambda^{D-1}$ being positive plastic compliance moduli, and $\hat{\mathbf{M}}^{i}$ and $\hat{\mathbf{N}}^{i}$ being pairs of yield and flow directions associated with each contributing mechanism.

Such a multi-mechanism formulation is well recognized in the field of polycrystalline plasticity dating back to seminal works by Taylor (1938), Bishop and Hill (1951), Bishop and Hill (1951), with its application to material plasticity and geomechanics further explored in studies such as Koiter (1953), Loret (1990), NematNasser (1983) and Anandarajah (2008). Moreover, the analysis in Kuhn and Daouadji (2018) has shown the appropriateness of such multi-mechanism models in capturing the plastic flow in granular media under coaxial conditions. As an extension to Kuhn and Daouadji (2018), the consequences pertaining to non-coaxial effects are analyzed in this study.

For the 2D cases considered in this study, a two-term form is adopted for the plastic constitutive tensor representing a doublemechanism plastic flow such that:

$\mathbb{C}=\Lambda^{1} \hat{\mathbf{N}}^{1} \hat{\mathbf{M}}^{1}+\Lambda^{2} \hat{\mathbf{N}}^{2} \hat{\mathbf{M}}^{2}$

The multi-mechanism expression adopted here differs from spectral (or singular value) decomposition representation, in that Eqs. 6 and 7 do not require orthonormality of $\hat{\mathbf{M}}^{i}$ and $\hat{\mathbf{N}}^{i}$ matrices. Instead, the first term on the right hand side of Eq. 7 is assumed to represent conventional coaxial plasticity, while the second term captures the additional non-coaxial part of plastic strain rate.

\subsection{Tensor decomposition for non-coaxiality}

To describe the non-coaxiality effects arising from rotation of principal stress directions, we propose (for the 2D case considered here) a decomposition of stress and strain tensors into so-called "in-plane" (denoted by $\pi$ ) and "out-of-plane" (denoted by $\rho$ ) parts, with the latter denoting the non-coaxial part. A more comprehensive formulation based on a representation theorem is recently presented in Li and Dafalias (2020) for general non-coaxial plastic flow in 3D cases. In this respect, we link coaxial plasticity to inplane parts and associate any deviation from it (non-coaxiality) to the out-of-plane ones. Detailed mathematical and graphical representations of these ideas are provided in Appendix A. 
In brief, for a given state of stress $\boldsymbol{\sigma}$ and an arbitrary second order tensor $\mathbf{A}$, the latter can be decomposed into its in-plane and out-of-plane parts such that $\mathbf{A}=\mathbf{A}^{\pi}+\mathbf{A}^{\rho}$ where $\mathbf{A}^{\pi}$ is coaxial with $\boldsymbol{\sigma}$ while $\mathbf{A}^{\rho}$ is non-coaxial with $\boldsymbol{\sigma}$, also satisfying $\mathbf{A}^{\rho}: \boldsymbol{\sigma}=0$. It is worth noting here that while $\mathbf{A}^{\rho}$ is orthogonal to $\boldsymbol{\sigma}$, orthogonality does not imply non-coaxiality. This is clear from the fact that the decomposition described here allows for the in-plane part of A to also have a part that is orthogonal to $\boldsymbol{\sigma}$. For the simple case of proportional loading (isobaric or biaxial) where the principal directions of stress (before applying the probes) are chosen as $x$ and $y$ axes, the decomposition of the stress (and strain) rate is straightforward:

$$
\begin{gathered}
\dot{\boldsymbol{\sigma}} \\
{\left[\begin{array}{cc}
\dot{\sigma}_{x x} & \dot{\sigma}_{x y} \\
\dot{\sigma}_{x y} & \dot{\sigma}_{y y}
\end{array}\right]=\left[\begin{array}{cc}
\dot{\sigma}_{x x} & 0 \\
0 & \dot{\sigma}_{y y}
\end{array}\right]+\left[\begin{array}{cc}
0 & \dot{\sigma}_{x y} \\
\dot{\sigma}_{x y} & 0
\end{array}\right]}
\end{gathered}
$$

Therefore, in this study we will use $\dot{\sigma}_{x y}$ (or its increment $d \sigma_{x y}$ ) to represent the out-of-plane stress rate. In this case, the unit tensor $\hat{\rho}$ denoting the direction of out-of-plane stress and strain is collinear with $\dot{\boldsymbol{\sigma}}^{\rho}$ in Eq. 8. For more general cases, such as simple shear, where the principal directions of the current stress continuously rotate, the appropriate variable $\dot{\boldsymbol{\sigma}}^{\rho}$ should be used based on the general decomposition described in Appendix A. Note that for 3D cases, both the in-plane and the out-of-plane spaces occupy higher dimensions, and as such, a more detailed decomposition is required to generalize the non-coaxial formulation, see also Li and Dafalias (2020).

In line with non-coaxial plastic flow, the plastic strain rate can thus be decomposed into its in-plane and out-of-plane parts such that $\dot{\boldsymbol{\varepsilon}}^{p}=\dot{\boldsymbol{\varepsilon}}^{p, \pi}+\dot{\boldsymbol{\varepsilon}}^{p, \rho}$, where $\dot{\boldsymbol{\varepsilon}}^{p, \pi}$ is coaxial with $\boldsymbol{\sigma}$ while the noncoaxial part is $\dot{\boldsymbol{\varepsilon}}^{p, \rho}$ (which also satisfies $\dot{\boldsymbol{\varepsilon}}^{p, \rho}: \boldsymbol{\sigma}=0$ ).

Substitution of Eq. 7 into Eq. 4 allows us to identify the in-plane and out-of-plane plastic strain rate contributions in the flow rule equation, i.e.

$$
\begin{array}{rlccc}
\dot{\boldsymbol{\varepsilon}}^{p} & = & \dot{\boldsymbol{\varepsilon}}^{p, \pi} & + & \dot{\boldsymbol{\varepsilon}}^{p, \rho} \\
& = & \Lambda^{1} \hat{\mathbf{N}}^{1}\left\langle\hat{\mathbf{M}}^{1}: \dot{\boldsymbol{\sigma}}\right\rangle & + & \Lambda^{2} \hat{\mathbf{N}}^{2}\left(\hat{\mathbf{M}}^{2}: \dot{\boldsymbol{\sigma}}\right)
\end{array}
$$

Note that the Macauley brackets for distinguishing between loading and unloading directions have only been applied to the first (inplane) term. This is because the current stress state (coaxial with current fabric here) does not set a preferred direction for the outof-plane space, and as such, unlike the in-plane part, the bracket is not used for the out-of-plane term. For the simple case of isobaric loading, this indicates that a symmetry exists between $d \sigma_{x y}$ and $-d \sigma_{x y}$ in that both directions produce plastic loading, which, although not explicitly presented here, is nevertheless confirmed by our DEM simulations. The loading/unloading condition can be more complicated for higher dimensions and also for cases where the fabric and stress tensors are not coaxial (Fu and Dafalias, 2011).

It is worth noticing that the form of Eq. 9 also resembles the plastic flow formulation for coaxial 3D cases such as in Nicot and Darve (2007), where, instead of the decomposition into in-plane (coaxial) and out-of-plane (non-coaxial) parts, the plastic strain is decomposed into components within and normal to the Rendulic plane. Our results show that such deviations from conventional plasticity are not limited to 3D cases and are observed also for non-coaxial 2D cases.

The general form in Eq. 9 will be further elaborated using the DEM simulation results presented in the next section.

\section{DEM simulations}

Two-dimensional DEM simulations have been performed using the open-source YADE software package (Šmilauer et al., 2010), to investigate the response of granular samples to stress probes. The samples consists of 50,000 circular particles, with radii uniformly distributed between $r_{\min }$ and $r_{\max }$, where $r_{\max } / r_{\min }=2$. A linear elastic force-deformation model is adopted for all contacts, with equal stiffness $k^{c}$ in the directions normal and tangential to the contact. This contact stiffness is determined from $k^{c}=E_{r_{1}^{c}+r_{2}^{c}}^{2 r^{c} r_{2}^{c}}$ with $r_{1}^{c}$ and $r_{2}^{c}$ being the radii of two particles in contact, and $E$ a stiffness parameter. The elastic interaction model is complemented with a cohesionless Coulomb frictional criterion, with interparticle friction coefficient $\mu=0.577$, i.e. a friction angle of $30^{\circ}$.

Periodic boundary conditions have been employed in order to suppress the formation of large-scale shear bands. The initial sample is isotropically compacted until the specified hydrostatic confinement $p_{0}$ is attained. In this compaction stage, the interparticle friction coefficient $\mu$ is set to zero to achieve a dense state. The confining stress, $p_{0}$, and the stiffness parameter $E$ are chosen such that the contact deformation is small relative to average particle size, since $\frac{p_{0}}{E \bar{r}}=5 \times 10^{-3}$ with $\bar{r}$ being the average particle radius. The two-dimensional porosity of the resulting dense sample equals 0.143 .

Overall strains are imposed by applying a velocity gradient to the particles along the periodic boundaries, and the stress is controlled via appropriate servo-control mechanisms. The average stress tensor of the assembly is determined from the micromechanical expression for the stress in terms of interparticle force vectors and branch vectors (Bagi, 1996; Weber, 1966).

After reaching conditions of static equilibrium under the confining pressure, the sample is loaded deviatorically under isobaric ( $p$ constant) conditions where the normal stress in the vertical $y$ direction $\left(\sigma_{y y}\right)$ is increased, while the hydrostatic stress, $p=\left(\sigma_{x x}+\sigma_{y y}\right) / 2$ is kept constant by adjusting the horizontal stress $\left(\sigma_{x x}\right)$ accordingly. No shear stress is applied in the reference coordinates, and hence, the principal directions of the stress tensor (aligned with horizontal and vertical directions) remain unchanged. The general stress-strain and volumetric responses are presented in Fig. 1, showing typical trends expected for an initially dense sample. In this study, extensive strains are considered positive.

As the deviatoric load is increased, the state of the assembly is stored at deviatoric stress ratios of $q / p=0.15,0.30$, and 0.40 , as indicated by black circles in Fig. 1(a). The fabric anisotropy, as defined in Rothenburg and Bathurst (1989), is found to be $0.034,0.0928$, and 0.160 , respectively. The stress probing procedure is then performed by starting from each of the three stored states.

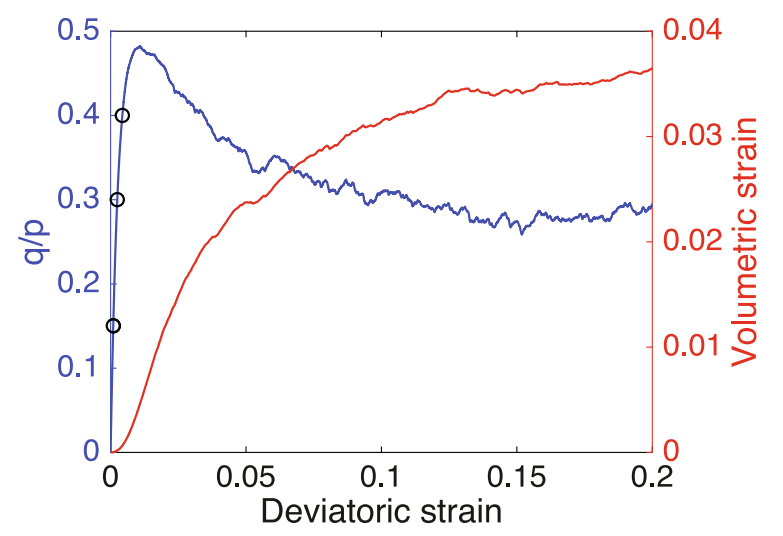

Fig. 1. Stress-strain and volumetric response of the isobaric test. The sampling stress states for probing are identified by black circles on the stress-strain curve. 


\subsection{Stress probing}

The stress probing method (Gudehus, 1979) is adopted here to investigate the incremental response of the granular media. The technique involves applying stress increments $d \boldsymbol{\sigma}$ along different "directions" in stress space while the magnitude of the stress increment $|d \boldsymbol{\sigma}|=d \sigma_{\text {probe }}$ is kept sufficiently small and equal for all probes.

In order to investigate the effect of non-coaxiality between the current stress state and the stress increment, a generalized stressprobing procedure is adopted here that differs from conventional probing in that the applied stress probe $d \boldsymbol{\sigma}$ contains out-of-plane shear components and thus is not necessarily coaxial with the current stress state $\boldsymbol{\sigma}$.

Adopting the vector representation for symmetric second-order tensors given in Eq. 3, the stress increment tensors $d \boldsymbol{\sigma}$ can be visualized in three-dimensional space as a sphere with radius $d \sigma_{\text {probe, }}$, as shown in Fig. 2(a). Due to symmetry considerations, only half of a sphere needs to be covered. For the current study the space of possible probes has been spanned by 151 stress probes along different directions: 25 probes for each of six azimuthal angles that correspond to a specific magnitude of the out-of-plane stress increment, as well as one probe with zero in-plane stress increment.

The magnitude of stress probes $d \sigma_{\text {probe }}$ should be kept sufficiently small such that the stress-strain response for each probe can be considered to be linear, which in turn ensures the firstorder homogeneity of the strain response with respect to the magnitude of the stress probes. The issue of the choice of the magnitude of the stress probe has been discussed in Froiio and Roux (2009) and Pouragha et al. (2019). Here, a relative stress probe size of $d \sigma_{\text {probe }} / p=10^{-2}$ has been adopted, based on a trial-and-error approach. While not presented here, direct inspection of the stress-strain response for each probe confirmed the required linearity of the response within the range of the employed stress probe magnitude.

\subsection{Elastic and plastic strains}

The decomposition of strain into its elastic and plastic components from results of frictional DEM simulations is not trivial. Due to the continuous evolution of the microstructure, loading/unloading cycles are erroneous in that irreversible mechanisms are observed to occur during load reversal (Pouragha and Wan, 2018; Pouragha et al., 2019). Numerical investigations have shown that an accurate estimate of the elastic deformation can be obtained when the applied stress path is repeated with frictional sliding being suppressed by the use of an artificially large interparticle friction coefficient $\mu$ (Pouragha and Wan, 2018; Pouragha et al., 2019). This latter method is also adopted in the present study, and as such, each stress probe involves two DEM simulations: (i) with the actual friction coefficient, giving the total strain response $\dot{\boldsymbol{\varepsilon}}$, and (ii) with artificially large interparticle friction coefficient $\mu$, giving the elastic strain response $\dot{\boldsymbol{\varepsilon}}^{e}$.

The plastic strain response $\dot{\boldsymbol{\varepsilon}}^{p}$ is then obtained from the additive decomposition of total strain increments into elastic and plastic strain increments, i.e.

$\dot{\boldsymbol{\varepsilon}}=\dot{\boldsymbol{\varepsilon}}^{e}+\dot{\boldsymbol{\varepsilon}}^{p}$

It has also been verified that the elastic response at each stress state conforms to a constant elastic (compliance) tensor, implying incrementally linear elastic responses. The elastic and plastic strain response envelopes obtained as such are visualized in Fig. 2(b).

\section{Analysis of probe responses}

We now turn our attention to the analysis of plastic strain response to stress probing, and specifically to the insights that they provide for further elaboration of the general plastic flow expression in Eq. 9. In order to avoid overcrowding the figures, the results are explicitly visualized only for the case at $q / p=0.3$. Given the scope of the study, the analyses are focused on the continuum (macroscopic) scale and, relevant though they are, the micromechanical aspects are not discussed here. Future studies are called for to investigate such micromechancial aspects of non-coaxial loading in detail.

As anticipated, the DEM simulation results given in Fig. 2(b) show a directional dependence of the plastic response on the stress increment direction. A formal analysis is presented in Appendix B where it is demonstrated that the plasticity relation is indeed incrementally nonlinear in nature with the plastic modulus (hence variables in Eq. 7) depending on loading direction.

Fig. 3 visualizes the direction of the plastic strain increment for each stress probe by superimposing both stress and strain spaces. As expected, Fig. 3(b) shows that in the absence of out-of-plane stress increments $\left(d \sigma^{\rho}=d \sigma_{x y}=0\right)$, the plastic flow attains no out-of-plane part ( $\left.d \varepsilon^{p, \rho}=d \varepsilon_{x y}^{p}=0\right)$. A single plastic flow direction is also realized for these probes. Both of these observations are consistent with the results from previous studies on conventional in-plane probing (Bardet, 1994; Calvetti et al., 2003; Froiio and Roux, 2009; Wan and Pinheiro, 2014). This indicates that the second term in Eq. 9 vanishes if $d \boldsymbol{\sigma}^{\rho}=\boldsymbol{0}$, which in turn means that $\hat{\mathbf{M}}^{2}: d \boldsymbol{\sigma}^{\pi}=0$. As such, for the $2 \mathrm{D}$ cases considered in this study,

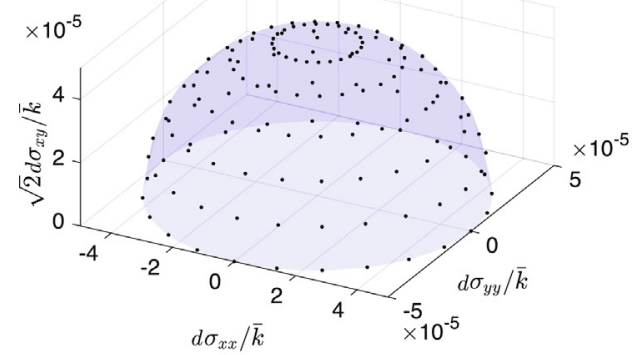

(a) Stress probe

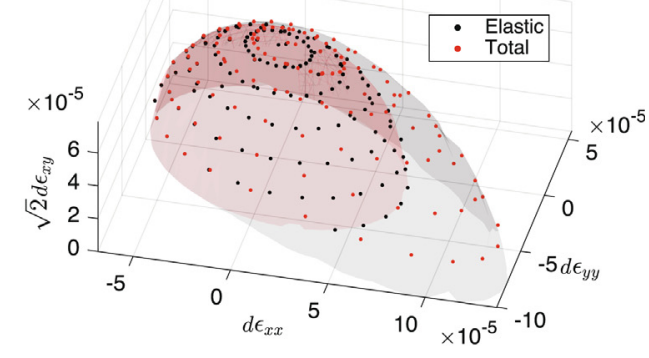

(b) Strain response

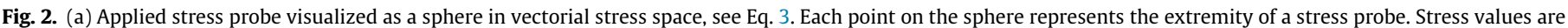

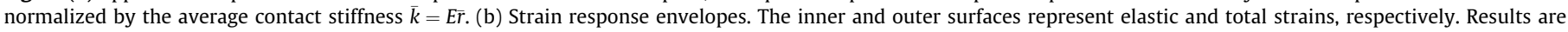
shown for the probing at $q / p=0.3$. 

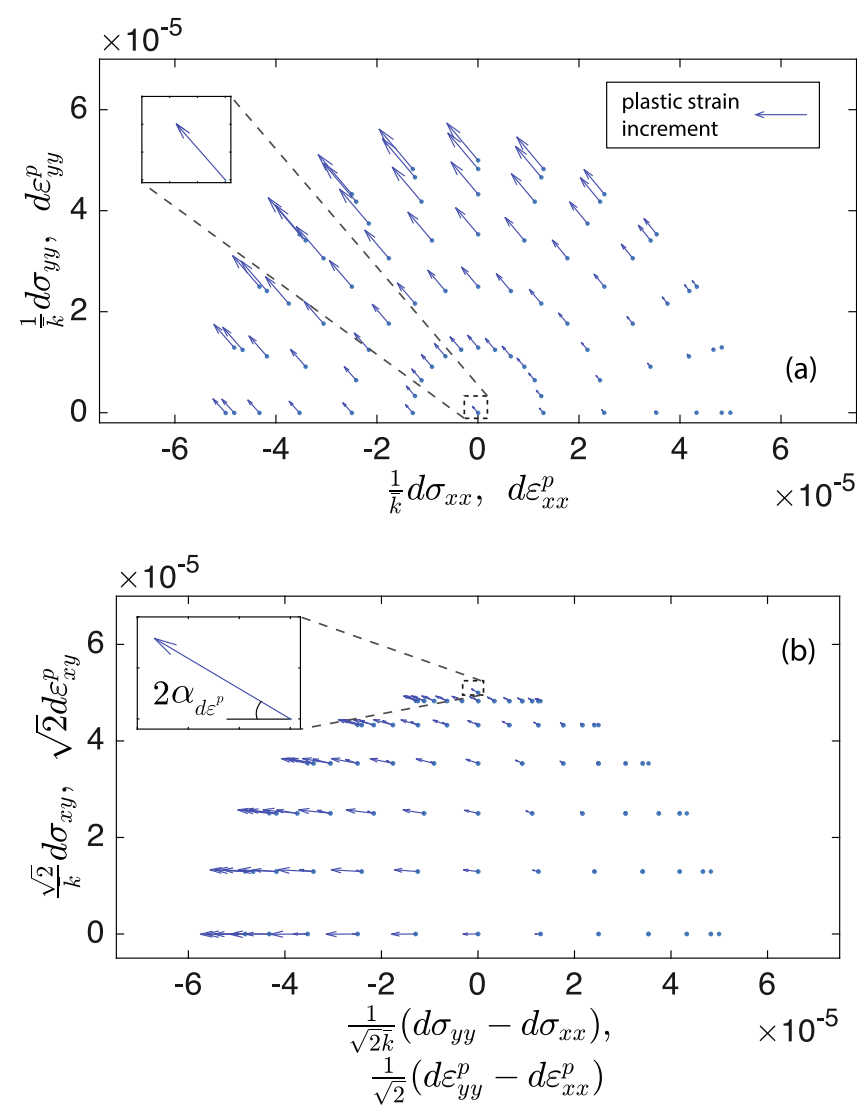

Fig. 3. Plastic flow direction visualized for all probes for the sample at $q / p=0.30$. (a) In-plane plastic flow visualized by the projection on the $x-y$ plane. (b) Out-ofplane response visualized by projection on the deviatoric plane with unit normal $\left\{\frac{1}{\sqrt{2}}, \frac{1}{\sqrt{2}}, 0\right\}$. The starting point of arrows is given by the stress probe values, $\left\{d \sigma_{x x}, d \sigma_{y y}, d \sigma_{x y}\right\}$, while the plastic strain increment $\left\{d \varepsilon_{x x}^{p}, d \varepsilon_{y y}^{p}, d \varepsilon_{x y}^{p}\right\}$ determines the components of the arrow vectors. Additional $\sqrt{2}$ factors are included in subfigure (b) for the projected data to fall along circular contours. Insets in sub-figure (a) and (b) show the zoomed view of the single shear-only probe.

$\hat{\mathbf{M}}^{2}$ is found to be proportional to $\hat{\boldsymbol{\rho}}$ which defines the direction of out-of-plane stress (and strain) rates, as explained in Appendix A.

The current study goes beyond the previous numerical simulations of probing, such as Bardet (1994), Calvetti et al. (2003), Froiio and Roux (2009), Wan and Pinheiro (2014), by also considering non-coaxial stress probes. Therefore, additional important observations have been made upon detailed examination of the data in Fig. 3, as listed in the following. While the results are only visualized for the case with $q / p=0.3$, the main observations below hold for all the probings carried out in this study.

\section{Non-coaxiality:}

Fig. 3(b) shows that, as the out-of-plane part of the stress probe $\left(d \sigma_{x y}\right)$ increases, the plastic flow direction deviates from the horizontal (in-plane) direction in a systematic manner. This non-zero out-of-plane plastic flow indicates the non-coaxiality of the plastic response with respect to the current stress, which further means that the second term in Eq. 9, and hence $\Lambda^{2}$, is non-zero. This is demonstrated more clearly by assigning a direction angle to the out-of-plane part of stress and plastic strain increments, $\alpha_{d \sigma}$ and $\alpha_{d \varepsilon^{p}}$ respectively, defined as Gutierrez et al. (1991):

$$
\begin{aligned}
& \alpha_{d \sigma}=\frac{1}{2} \tan ^{-1}\left(\frac{2 d \sigma_{x y}}{d \sigma_{y y}-d \sigma_{x x}}\right), \\
& \alpha_{d \varepsilon^{p}}=\frac{1}{2} \tan ^{-1}\left(\frac{2 d \varepsilon_{x y}^{p}}{d \varepsilon_{y y}^{p}-d \varepsilon_{x x}^{p}}\right)
\end{aligned}
$$

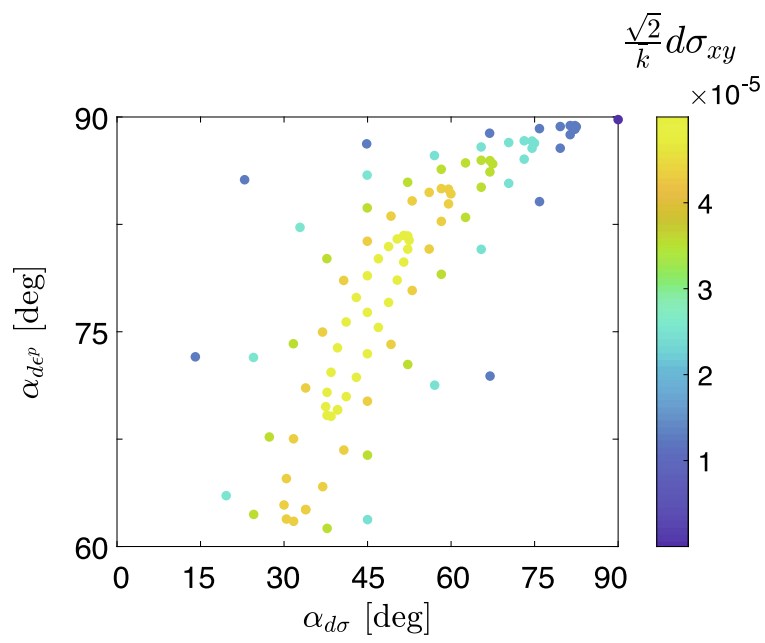

Fig. 4. Variation of plastic strain response direction $\alpha_{d z^{p}}$ with stress probe direction $\alpha_{d \sigma}$ for the case with $q / p=0.30$. The data points are color-coded based on the magnitude of out-of-plane stress increment.

The variations of these angles are presented in Fig. 4. The single dark data point at $\left\{90^{\circ}, 90^{\circ}\right\}$ represents all the in-plane probes, i.e. horizontal arrows at $d \sigma_{x y}=0$ in Fig. 3(b). It is clear from Fig. 4 that both stress probe direction, $\alpha_{d \sigma}$, and plastic strain response direction, $\alpha_{d \varepsilon^{p}}$, deviate simultaneously from $90^{\circ}$, indicating that stress probes with out-of-plane parts induce outof-plane plastic strain parts. Notice that even for relatively small values of out-of-plane stress increment, $d \sigma_{x y}$ (darker points), considerable deviations from $90^{\circ}$ are observed for $\alpha_{d \sigma}$ and $\alpha_{d \varepsilon^{p}}$, which is due to the definitions adopted in Eq. 11 whereby the denominator can also become small for these probes.

2. Coupling between in-plane and out-of-plane parts:

While in-plane stress probes do not produce out-of-plane plastic strains (previous observation), the opposite does not hold true. The out-of-plane part of the stress probe $\left(d \sigma^{\rho}=d \sigma_{x y}\right) \underline{\text { does }}$ contribute to in-plane plane plastic strain $d \boldsymbol{\varepsilon}^{p, \pi}$. This is clearest for the single stress probe with zero in-plane part shown in the insets in Fig. 3(a) and (b).

Therefore, recalling that the first term in Eq. 9 describes the inplane plastic strain, it transpires that $\hat{\mathbf{M}}^{1}: d \boldsymbol{\sigma}^{\rho} \neq 0$. This means that $\hat{\mathbf{M}}^{1}$ (representing the gradient of the yield function in conventional elasto-plasticity) is not entirely in-plane and involves an out-of-plane part. The possibility of such a dependency is also envisaged in constitutive models that consider kinematic hardening with a back-stress tensor that is not necessarily coaxial with the current stress (Dafalias and Feigenbaum, 2011).

3. Uniqueness of in-plane flow direction:

As a crucial observation, Fig. 3(a) shows that, regardless of the out-of-plane stress increment $\left(d \sigma^{\rho}=d \sigma_{x y}\right)$, the in-plane part of plastic strain flow follows a single direction. This is more conclusively visualized in Fig. 5 where the in-plane parts of plastic flow are shown to collapse along a single straight line. Data points are color-coded based on the magnitude of the out-ofplane stress increment. It is worth noting that, unlike observation (2), the results in Fig. 5 include all the probes.

This observation is consistent with our earlier assumption in Eq. 9 where the second additional term is assumed to capture only the out-of-plane plastic strain. As such, similar to $\hat{\mathbf{M}}^{2}$, the plastic strain direction $\hat{\mathbf{N}}^{2}$ also turns out to be normal to the plane of $\pi$, 


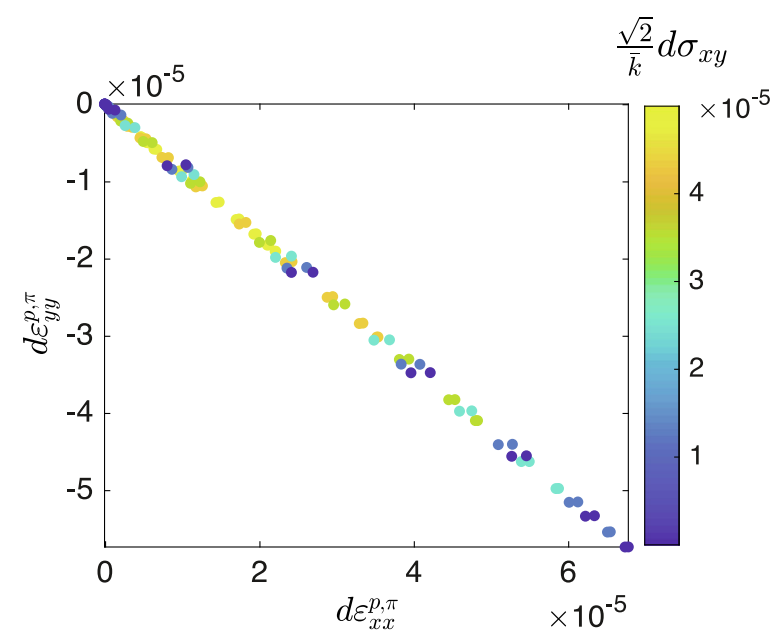

Fig. 5. In-plane plastic strains $d \varepsilon^{p, \pi}$ for the case with $q / p=0.30$.

i.e. $\hat{\mathbf{N}}^{2}=\hat{\boldsymbol{\rho}}$.

Substituting the relations $\hat{\mathbf{M}}^{2}=\hat{\mathbf{N}}^{2}=\hat{\boldsymbol{\rho}}$ into Eq. 9 yields:

$\dot{\boldsymbol{\varepsilon}}^{p}=\Lambda^{1} \hat{\mathbf{N}}^{1}\left\langle\hat{\mathbf{M}}^{1}: \dot{\boldsymbol{\sigma}}\right\rangle+\Lambda^{2} \hat{\boldsymbol{\rho}}(\hat{\boldsymbol{\rho}}: \dot{\boldsymbol{\sigma}})=\Lambda^{1} \hat{\mathbf{N}}^{1}\left\langle\hat{\mathbf{M}}^{1}: \dot{\boldsymbol{\sigma}}\right\rangle+\Lambda^{2} \dot{\boldsymbol{\sigma}}^{\rho}$

where $\dot{\boldsymbol{\sigma}}^{\rho}=\hat{\boldsymbol{\rho}}(\hat{\boldsymbol{\rho}}: \dot{\boldsymbol{\sigma}})$ is the out-of-plane part of the plastic strain rate, see Appendix A.

It is worth mentioning here that the transition from Eq. 9 to 12 is not readily applicable to 3D cases where the direction of out-ofplane stresses and strains is not fixed for a given plane of $\pi$. For instance, for the 3D case with only normal initial stresses, the out-of-plane stress increments can assume different proportions of three independent shear stress components. The recent study in Li and Dafalias (2020) outlines how a representation theorem can be used to generalize the decomposition Eq. 9 to 3D cases. Nevertheless, Eq. 9 can be considered as an appropriate primary candidate for 3D cases as well, since no coupling among the various dimensions of out-of-plane stress increments can be readily envisaged. Such a claim, however, needs careful examination and thorough justification which falls beyond the scope of this study.

The model parameters in Eq. 12, including $\Lambda^{1}, \hat{\mathbf{M}}^{1}, \hat{\mathbf{N}}^{1}, \Lambda^{2}, \hat{\mathbf{M}}^{2}$, and $\hat{\mathbf{N}}^{2}$, can now be fitted for each of the three sets of probing data (with initial deviatoric stress ratio of $q / p=0.15,0.3$, and 0.4 ) via the following procedure;

- In-plane flow direction $\hat{\mathbf{N}}^{1}$ is found by fitting a straight line to the in-plane plastic strain parts, $d \varepsilon^{p, \pi}$, similar to Fig. 5.

- Yield direction $\hat{\mathbf{M}}^{1}$ and the compliance modulus $\Lambda^{1}$ are computed through a least-squares scheme by fitting the magnitude of in-plane plastic strain in terms of stress probes.

- Out-of-plane compliance modulus $\Lambda^{2}$ is found by fitting a straight line to the $d \varepsilon^{p, \rho}$ vs. $d \sigma^{\rho}$ trend.

The values for calibrated parameters are given in Table C.1. Below we analyze each of these variables separately in the following subsections.

\subsection{In-plane flow direction $\hat{\mathbf{N}}^{1}$}

From the results in Fig. 5, a unique direction for in-plane plastic flow $\left(\hat{\mathbf{N}}^{1}\right)$ is recognized which is independent of loading direction $\hat{\dot{\sigma}}$. This implies that the conventional single-mechanism plasticity theory holds for the in-plane response of 2D cases. This observation, however, will not hold for 3D cases as shown before via coaxial probing studies (Kuhn and Daouadji, 2018; Nicot and Darve, 2007; Wan and Pinheiro, 2014; Harthong and Wan, 2013).

\subsection{Yield direction $\hat{\mathbf{M}}^{1}$ and compliance modulus $\Lambda^{1}$}

Fig. 6 compares the increments of in-plane plastic strain increments with the term $\hat{\mathbf{N}}^{1}\left\langle\hat{\mathbf{M}}^{1}: \dot{\boldsymbol{\sigma}}\right\rangle$ in Eq. 12. The slope of the trend gives the value of $\Lambda^{1}$. It is clear that although a straight line perfectly fits the data points pertaining to in-plane stress probes (dark blue points with $d \sigma_{x y}=0$ ), systematic deviations are observed in the presence of out-of-plane stress increments (lighter colored points), which is reflective of the incrementally nonlinear nature of the plastic response as demonstrated in Appendix B. Therefore, one can enhance the accuracy of predictions at the cost of including additional model parameters, by assuming an incrementally nonlinear form in which the variable $\Lambda^{1}$ depends on the stress probe direction. The matter is, however, not explored further here since the deviations are small.

\subsection{Out-of-plane compliance modulus $\Lambda^{2}$}

The variation of the out-of-plane plastic strain with the out-ofplane stress increment is given in Fig. 7. Based on the formulation in Eq. 12, a constant value for $\Lambda^{2}$ will lead to a linear relationship, shown by the straight dashed black line, whose slope is $\Lambda^{2}$. However, the significant scatter of the data points clearly indicates the dependency of $\Lambda^{2}$ on loading direction, hence incremental non-linearity. This is expected based on the results in Appendix $\mathrm{B}$ where the deviation from incremental linearity is shown to be more significant for the out-of-plane strains.

In this case, a correlation analysis with different variables has shown that the incremental non-linearity can be modelled by assuming a dependence of $\Lambda^{2}$ on the term $\left\langle\hat{\mathbf{M}}^{1}: \hat{\dot{\boldsymbol{\sigma}}}\right\rangle$. The dependency is visualized via color-coded contour lines of constant $\left\langle\hat{\mathbf{M}}^{1}: \hat{\boldsymbol{\sigma}}\right\rangle$ in Fig. 7 where relatively linear trends are observed for

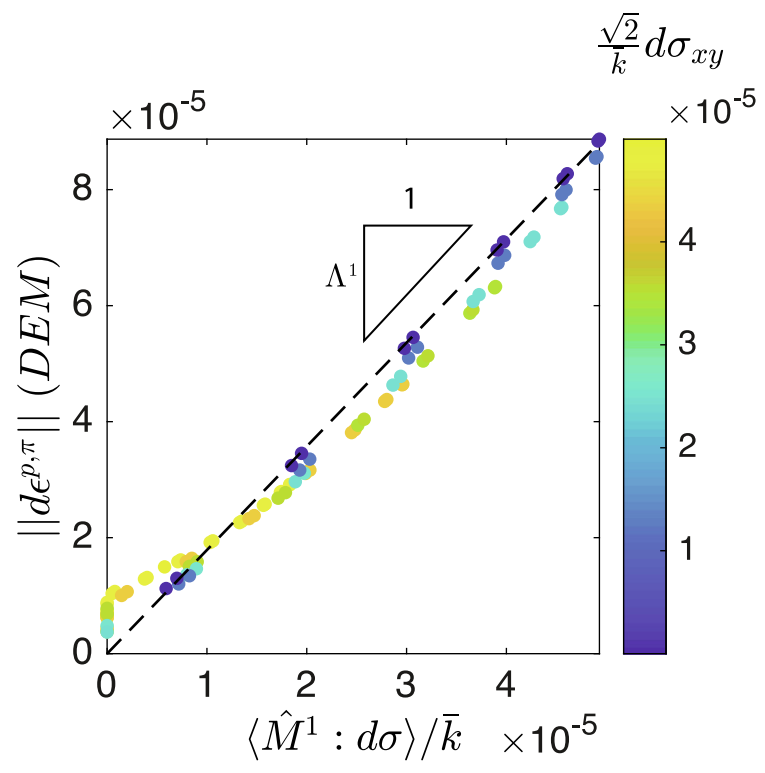

Fig. 6. In-plane plastic strain increment vs the term $\left\langle\hat{\mathbf{M}}^{1}: d \boldsymbol{\sigma}\right\rangle$ for the case with $q / p=0.30$. The slope of the trend is $\Lambda^{1}$. The values are color-coded based on their out-of-plane stress increments. $\bar{k}$ is the average contact stiffness. 


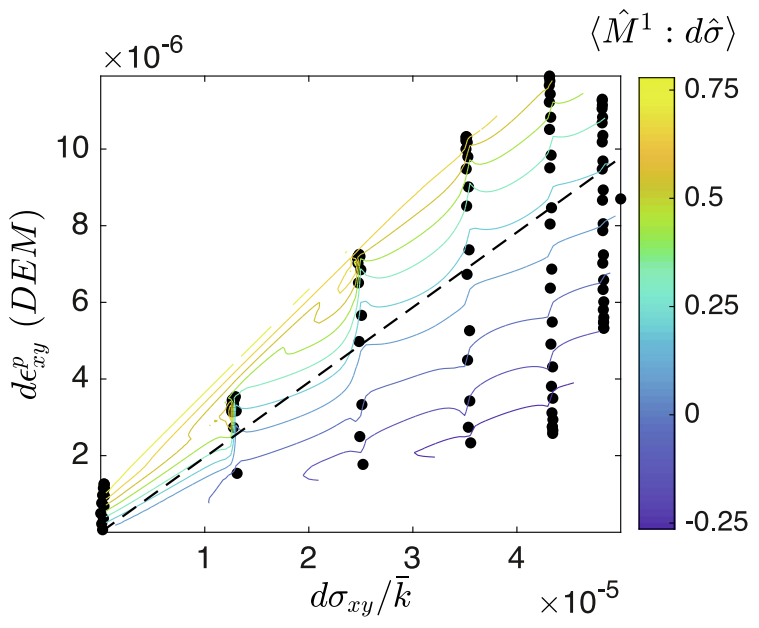

Fig. 7. Variation of out-of-plane plastic strain vs. out-of-plane stress increments for the case with $q / p=0.30$. The colored lines represent contours of constant $\left\langle\hat{\mathbf{M}}^{1}: \hat{\boldsymbol{\sigma}}\right\rangle$ indicating incremental non-linearity of the plastic response. The dashed black line represents the fitted linear variation associated with a constant value of $\Lambda^{2}$.

cases with constant $\left\langle\hat{\mathbf{M}}^{1}: \hat{\boldsymbol{\sigma}}\right\rangle$, verifying the appropriateness of the chosen variable. However, a more involved fitting attempt is avoided here in favor of clarity of the main ideas.

\section{Discussion}

The analysis of the probing results in the previous section shows that the expression in Eq. 12 encapsulates the salient characteristics of plastic flow in granular materials under non-coaxial loading conditions. The developed plastic flow formulation differs from conventional elasto-plasticity theory in two main aspects: (1) The additional term associated with $\Lambda^{2}$ implies the existence of two separate mechanisms and their respective yield and flow directions, which leads naturally to non-coaxial responses, and;

(2) The yield direction in the first term $\hat{\mathbf{M}}^{1}$ is not necessarily coaxial with the current stress.

By separating different prevalent mechanisms, the formulation in Eq. 12 also provides a direct measure for the significance of the non-coaxiality effect via the ratio between the compliance moduli $\Lambda^{1}$ and $\Lambda^{2}$. The variation of this ratio with the deviatoric stress ratio (current stress state of the material) is provided in Fig. 8 indicating that ignoring the non-coaxial terms can potentially lead to $\sim 20 \%$ of error in plastic deformations. However, the significance

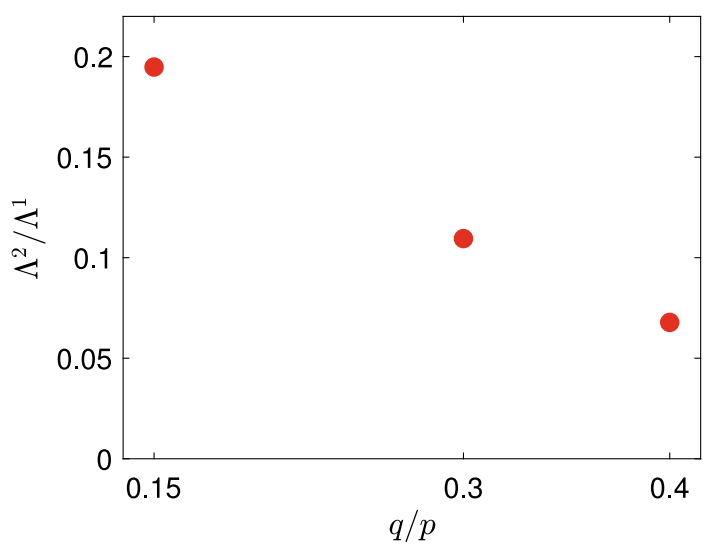

Fig. 8. Variation of the significance of non-coaxiality, $\Lambda^{2} / \Lambda^{1}$, with deviatoric stress ratio, $q / p$. of the effects decreases as the stress state of material approaches stress peak since the ratio of $\Lambda^{2} / \Lambda^{1}$ decreases with increasing deviatoric stress ratio. This indicates that although initially multiple plastic mechanisms contribute to the behaviour of granular materials, a single mechanism emerges to dominate the response as the stress peak is approached. This finding echos previous observations in Yamada and Ishihara (1979), Ochiai and Lade (1983), Lade et al. (2009), and Rycroft et al. (2009) where a similar decrease in the effect of material anisotropy on plasticity and failure characteristics of granular media has also been reported.

Finally, the general characteristics of the developed model are compared with two previously developed models: the one by Tsutsumi and Hashiguchi (2005), and the double-shearing theory (Mehrabadi and Cowin, 1978). These two theories are selected for our comparison mainly because they are based on clear physical assumptions that can be checked without a full calibration of the model.

\subsection{Comparison to plasticity model by Hashiguchi and co-workers}

Although the final form of the plasticity formulation developed herein resembles that of Tsutsumi and Hashiguchi (2005), they differ in one key aspect, namely that the latter ignores the coupling between the in-plane and out-of-plane stress and strain. More specifically, the formulation adopted by Hashiguchi and coworkers assumes a priori that the out-of-plane stress increments cannot produce in-plane plastic strains, which is in contrast with our DEM stress probing results. The general basis provided by Eq. 9 , on the other hand, serves as a sound starting point whereby such couplings can be captured. In this case, the plasticity flow relation given by Eq. 12 reduces to the model by Hashiguchi and coworkers only if the tensor $\hat{\mathbf{M}}^{1}$ is assumed to be in-plane.

A more subtle difference also exists between the two approaches as the decomposition into in-plane and out-of-plane parts in Tsutsumi and Hashiguchi (2005) is based on coaxiality with $\hat{\mathbf{M}}^{1}$ and not with the current stress tensor $\boldsymbol{\sigma}$. The difference is, nevertheless, less significant, recalling that in Tsutsumi and Hashiguchi (2005) the two tensors are assumed to be coaxial.

\subsection{Comparison to double-shearing plasticity models}

An important class of non-coaxial plasticity models originates from the double-shearing theory developed by Spencer (1964), Mehrabadi and Cowin (1978), Mehrabadi and Cowin (1981), and elaborated later by such works as Harris (1995), Nemat-Nasser and Zhang (2002), Zhu et al. (2007), and Yu and Yuan (2006). A critical assessment of the model's application to plastic flow of cohesionless granular materials is given in Kruyt (1990).

The algebraic treatments made by Harris (1995) show that the double-shearing model also leads to plasticity expressions that resemble Eq. 12, with the difference that the out-of-plane part of stress increment is replaced with the equivalent stress rotation. Notwithstanding the similarities in final formulations, the double-shearing approach differs from our formulation in that it starts by assuming a fixed "angle of dilatancy" that describes the ratio between normal and tangential plastic strains along characteristic slip lines. ${ }^{1}$

Based on the calculation in Mehrabadi and Cowin (1978) the double-shearing theory predicts a proportional relation between components of plastic strain as follows:

\footnotetext{
1 The "angle of dilatancy", as defined in double-shearing theory, should not be confused with the common notion of dilatancy that measures the incremental ratio between volumetric and deviatoric plastic strains. The latter is not fixed in doubleshearing theory.
} 


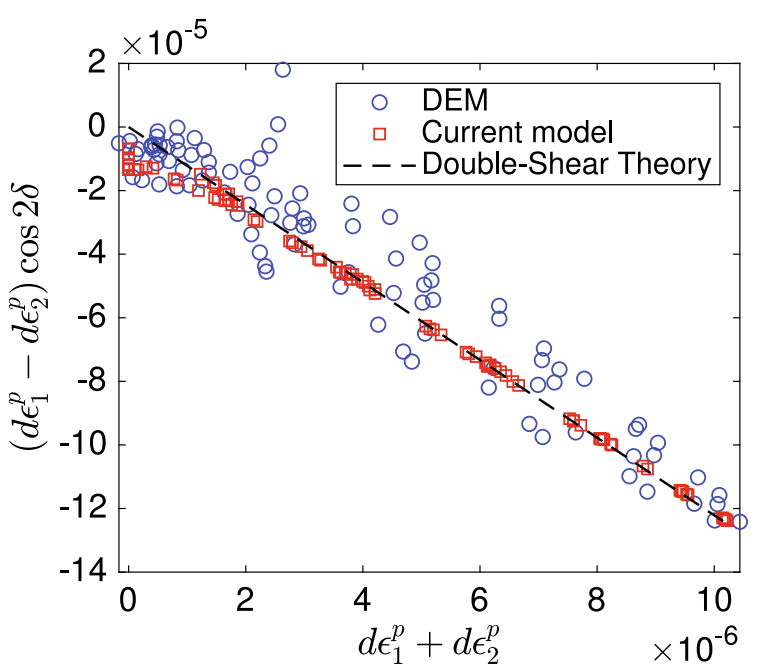

Fig. 9. Comparison between the predictions by the current model (Eq. 12), doubleshearing theory, and DEM simulation results for the case with $q / p=0.3$.

$d \varepsilon_{1}^{p}+d \varepsilon_{2}^{p}=A\left(d \varepsilon_{1}^{p}-d \varepsilon_{2}^{p}\right) \cos 2 \delta$

$\tan 2 \delta=\frac{2 d \varepsilon_{x y}^{p}}{d \varepsilon_{x x}^{p}+d \varepsilon_{y y}^{p}}$

where $d \varepsilon_{1}^{p}$ and $d \varepsilon_{2}^{p}$ are the principal values of plastic strain increment and $A$ is a constant that depends on internal friction and the angle of dilatancy. Compared to the formulation in Mehrabadi and Cowin (1978), the principal direction of stress is chosen as the reference here.

Fig. 9 illustrates the proportionality given by Eq. 13 alongside the DEM results and the prediction by the current model given by Eq. 12 . The comparison shows that despite their different points of departure, the current plastic flow model and the doubleshearing theory yield consistent predictions. The scatter of the DEM results compared to the two models is associated with incrementally nonlinear nature of the response ignored by both models.

It is also worth mentioning that, similar to the current model's prediction in Fig. 7, the double shearing model also predicts a proportional relation between out-of-plane stress and plastic strain increments.

\section{Conclusions}

The study investigates plastic flow of granular materials subjected to non-coaxial incremental loading. A decomposition of plastic flow and stress rate into in-plane and out-of-plane parts is proposed, with the latter pertaining to the parts that are not coaxial with current stress state. By considering multiple mechanisms contributing to the plastic strain, an extension to the classical elasto-plastic formulation is suggested that captures the noncoaxial plastic response via a second set of flow directions. An extensive set of 2D DEM simulations of stress probing, involving loading with out-of-plane parts, is then performed to assess the suitability of the proposed non-coaxial plastic formulation. The simulation results indicate the significance of the non-coaxial effects to be around $\sim 20 \%$, which decreases as the sample reaches the stress peak.

The simulation results indicate that, for all stress probes, the inplane part of plastic flow lies along a single line. More importantly, the results point towards a coupling effect between out-of-plane and in-plane stress and strain increments whereby the out-ofplane (non-coaxial) stress increments lead to in-plane plastic strains. As such, the yield direction associated with in-plane responses ( $\hat{\mathbf{M}}^{1}$ ) involves an out-of-plane part. This crucial coupling has been ignored in conventional elasto-plasticity and more elaborate non-coaxial models (Tsutsumi and Hashiguchi, 2005).

The DEM results also indicate the incrementally nonlinear nature of the plastic flow and potential dependency of the model coefficients on loading direction. While this effect was not explicitly considered in the formulations, suggestions have been provided for further extensions.

Finally, the model characteristics have been compared, qualitatively and quantitatively, with two previous models: the noncoaxial model by Tsutsumi and Hashiguchi (2005) and the double-shearing theory (Mehrabadi and Cowin, 1978), outlining their differences and the similarities. Interestingly, the developed formulation is observed to produce similar trends to that of double-shearing theory.

Although our numerical simulations have been limited to 2D cases, the basics of the formulation are developed in a general form that can serve as a starting point for further extensions to 3D cases. Future studies are also required to investigate the micromechanical nature of non-coaxial responses, examples of which can be found in Li and Yu (2015), Gao and Zhao (2017), Shi et al. (2020), Nicot and Darve (2007). In particular, cases can be considered where the contact fabric tensor is not coaxial with the stress tensor in order to determine which tensorial quantity, stress or fabric, describes the non-coaxial effects more appropriately. Along this vein, the general decomposition of 3D deviatoric tensors (based on a representation theorem) formulated in $\mathrm{Li}$ and Dafalias (2020) may provide proper tools for analyzing such generalized loading conditions.

\section{Declaration of Competing Interest}

The authors declare that they have no known competing financial interests or personal relationships that could have appeared to influence the work reported in this paper.

\section{Acknowledgments}

Research funding provided by the Natural Sciences and Engineering Research Council of Canada (RGPIN-2020-06480 and DGECR-2020-00411 held by M. Pouragha and RGPIN-2016-04086 held by R. Wan) is gratefully acknowledged.

\section{Appendix A. In-plane and out-of-plane decomposition}

A decomposition of symmetric, second-order tensors (or vectors in the vector representation of stress and strain according to Eq. 3) is adopted in this study in order to develop plasticity expressions in a canonical form. The method employed here is inspired by that given in Tsutsumi and Hashiguchi (2005). However, detailed but important differences exist between the two methods.

In the two-dimensional case, the subspace of non-spherical tensors with the same principal directions as $\boldsymbol{\sigma}$ is spanned by the two orthogonal unit tensors $\hat{\tau}$ and $\hat{\boldsymbol{\delta}}$. This subspace of coaxial, or "inplane", tensors is denoted by $\pi$ (not to be confused with the common notion of deviatoric $\pi$-plane) while its complementary subspace is called "out-of-plane", denoted by $\rho$. Therefore, an arbitrary tensor $\mathbf{A}$ can be decomposed into its in-plane and outof-plane parts, $\mathbf{A}^{\pi}$ and $\mathbf{A}^{\rho}$, as follows:

$$
\begin{gathered}
\mathbf{A}=\mathbf{A}^{\pi}+\mathbf{A}^{\rho} \\
\mathbf{A}^{\pi}=(\mathbf{A}: \hat{\boldsymbol{\tau}}) \hat{\boldsymbol{\tau}}+(\mathbf{A}: \hat{\boldsymbol{\delta}}) \hat{\boldsymbol{\delta}} \\
\mathbf{A}^{\rho}=\mathbf{A}-\mathbf{A}^{\pi}
\end{gathered}
$$


For the two-dimensional case with three degrees of freedom for stress (and strain) components, the direction of $\mathbf{A}^{\rho}$ is easily determined as the space orthogonal to the in-plane subspace. Denoted by $\hat{\boldsymbol{\rho}}$, the direction is selected as the cross product of $\hat{\tau}$ and $\hat{\boldsymbol{\delta}}$ when represented as vectors (see Eq. 3 ):

$\boldsymbol{\rho}=\left[\begin{array}{cc}-\sqrt{2} \sigma_{x y} & \sigma_{x x}-\sigma_{y y} \\ \sigma_{x x}-\sigma_{y y} & \sqrt{2} \sigma_{x y}\end{array}\right] \quad \hat{\boldsymbol{\rho}}=\frac{\boldsymbol{\rho}}{\|\boldsymbol{\rho}\|}$

with the orthogonal triad $\hat{\boldsymbol{\tau}}, \hat{\boldsymbol{\delta}}$ and $\hat{\boldsymbol{\rho}}$ forming a canonical basis. Note that the representation above is general to 2D cases such that $x$ and $y$ directions do not need to correspond to the principal directions of $\boldsymbol{\sigma}$. Extensions of such cross-products to the more general threedimensional case can be found in Szabó (2016).

Fig. A.1 shows a schematic illustration of the vector representation given by Eq. 3 for two-dimensional symmetric tensors, together with the decomposition of a stress increment tensor $d \boldsymbol{\sigma}$ into its in-plane and out-of-plane parts, $d \boldsymbol{\sigma}^{\pi}$ and $d \boldsymbol{\sigma}^{\rho}$ respectively for an arbitrary given stress state $\boldsymbol{\sigma}$. Note also that the formulation in Eq. 14 allows for $d \boldsymbol{\sigma}^{\pi}$ to have a part that is orthogonal to $\boldsymbol{\sigma}$. A general formulation of such a decomposition in threedimensional cases is suggested in Li and Dafalias (2020).

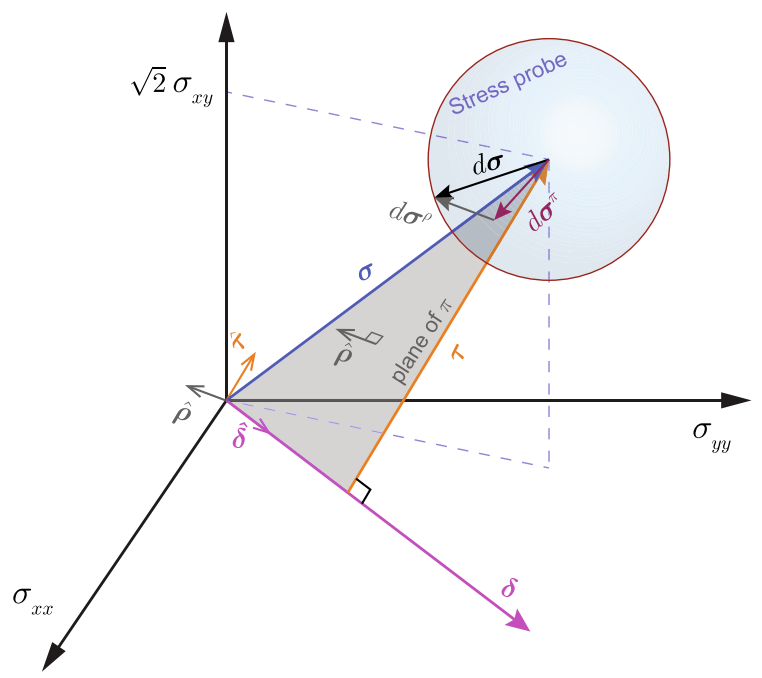

Fig. A.1. Visualisation of the decomposition of the stress rate tensor $\dot{\boldsymbol{\sigma}}$ into "inplane" and "out-of-plane" parts $\dot{\boldsymbol{\sigma}}^{\pi}$ and $\dot{\boldsymbol{\sigma}}^{\rho}$, respectively.

\section{Appendix B. Incremental non-linearity}

The incrementally nonlinear nature of the plastic response of the studied samples is briefly demonstrated here. Based on Eq. 4, an incrementally linear plastic modulus, $\mathbb{C}(\boldsymbol{\sigma})$, can be calculated by fitting a constant tensor to the plastic strain response envelope in terms of stress probes. The fitting is carried out using the leastsquares scheme. Fig. B.1 shows the deviations of the measured DEM data from those predicted by an incrementally linear relation with a constant compliance modulus $\mathbb{C}$. Data are color-coded based on the dimensionless magnitude of the out-of-plane stress increment $d \sigma_{x y} / \bar{k}$ where $\bar{k}$ is the average contact stiffness.

Clear deviations from the 1:1 dashed lines in Fig. B.1 indicate an incrementally nonlinear behaviour that cannot be captured with a constant plastic modulus. It is interesting to observe that the magnitude of deviation clearly correlates with the magnitude of the out-of-plane stress, which implies a correlation between the two phenomena of non-coaxiality and incremental non-linearity. Deeper interpretation of such an interrelation calls for more detailed future investigations that are outside the scope of this study.

\section{Appendix C. Calibrated model parameters}

The calibrated model parameters are given in a canonical frame of reference expressed by the orthonormal basis given by unit tensors (or vectors in the case of vector representation) $\{\hat{\boldsymbol{\delta}}, \hat{\boldsymbol{\tau}}, \hat{\boldsymbol{\rho}}\}$ which represent the directions of identity (hydrostatic stress), deviatoric stress, and out-of-plane stress tensors:

$\mathbf{A}=A_{\delta} \hat{\boldsymbol{\delta}}+A_{\tau} \hat{\boldsymbol{\tau}}+A_{\rho} \hat{\boldsymbol{\rho}}$

with the term $A_{\delta} \hat{\boldsymbol{\delta}}+A_{\tau} \hat{\tau}$ representing the in-plane projection of $\mathbf{A}$. Note that the representation based on Eq. 16 only applies to two-dimensional cases. For three-dimensional cases, more basis "vectors" are required to span the in-plane and out-of-plane spaces (Li and Dafalias, 2020).

The calibrated model parameters are given in Table C. 1 for the three stress ratios, $q / p$, considered in this study. The values of the compliance moduli $\Lambda^{1}$ and $\Lambda^{2}$ are non-dimensionalized by multiplying with the average contact stiffness $\bar{k}$.

The out-of-plane part of $\hat{\mathbf{N}}^{1}$ is zero by definition since it describes the in-plane plastic strain rates. Moreover, the ratio between $\widehat{N}_{\delta}^{1}$ and $\widehat{N}_{\tau}^{1}$ signifies the dilatancy associated with inplane parts of plastic strain increments.
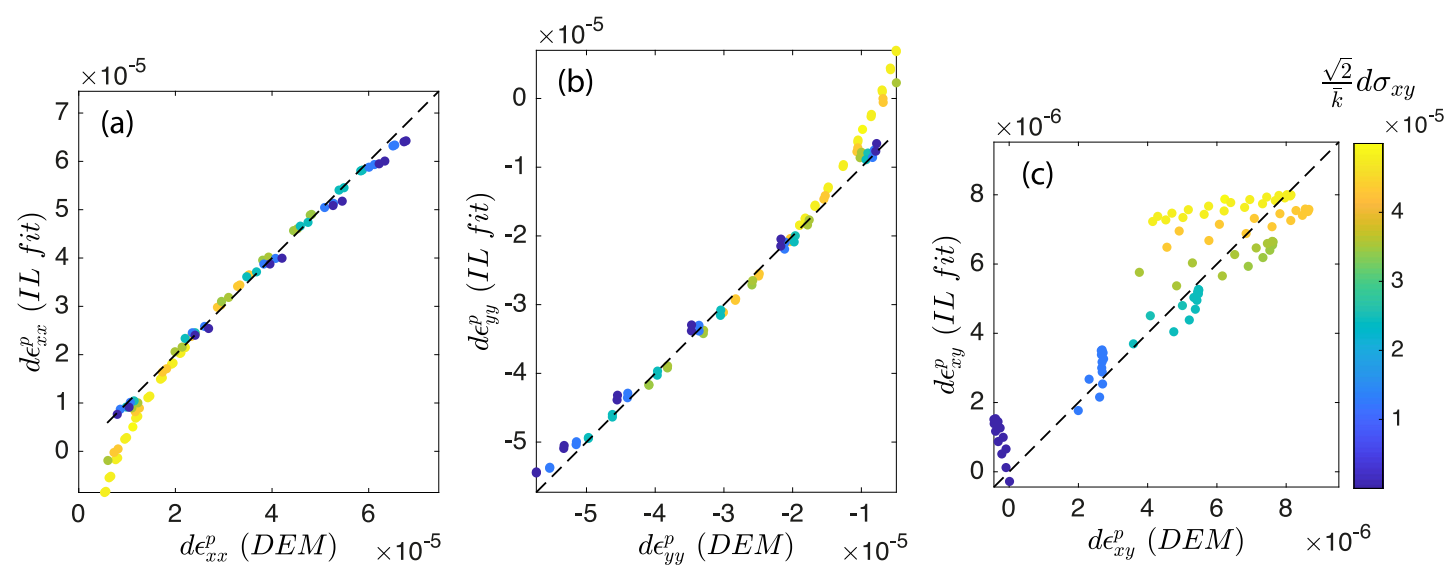

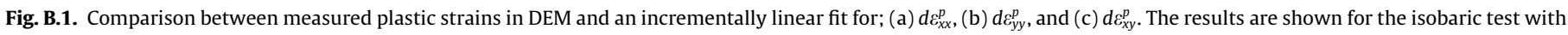
$q / p=0.3$. The deviations from $1: 1$ dashed lines indicate incremental non-linearity. The color mapping is the same for all sub-figures. 
Table C.1

Calibrated model parameters.

\begin{tabular}{|c|c|c|c|c|}
\hline \multicolumn{2}{|c|}{$q / p$} & 0.15 & 0.3 & 0.40 \\
\hline \multirow[t]{3}{*}{$\hat{\mathbf{M}}^{1}$} & $\hat{M}_{\delta}^{1}$ & 0.3462 & 0.3905 & 0.4209 \\
\hline & $\widehat{M}_{\tau}^{1}$ & 0.9178 & 0.9141 & 0.9046 \\
\hline & $\widehat{M}_{\rho}^{1}$ & 0.1946 & 0.1094 & 0.0678 \\
\hline \multirow[t]{3}{*}{$\hat{\mathbf{N}}^{1}$} & $\widehat{N}_{\delta}^{1}$ & 0.0208 & 0.0819 & 0.1476 \\
\hline & $\widehat{N}_{\tau}^{1}$ & 0.9998 & 0.9966 & 0.9890 \\
\hline & $\widehat{N}_{\rho}^{1}$ & 0 & 0 & 0 \\
\hline \multicolumn{2}{|c|}{$\Lambda^{1} \bar{k}$} & 0.3807 & 1.7873 & 6.1737 \\
\hline \multicolumn{2}{|c|}{$\Lambda^{2} \bar{k}$} & 0.0742 & 0.1957 & 0.4188 \\
\hline
\end{tabular}

\section{References}

Hill, R., 1998. The Mathematical Theory of Plasticity. Oxford University Press.

Ziegler, H., 1970. Proof of an orthogonality principle in irreversible thermodynamics. Z. Angew. Math. Phys. ZAMP 21 (6), 853-863.

Houlsby, G.T., Puzrin, A.M., 2007. Principles of Hyperplasticity: An Approach to Plasticity Theory based on Thermodynamic Principles. Springer Science \& Business Media.

Kuhn, M.R., Daouadji, A., 2018. Multi-directional behavior of granular materials and its relation to incremental elasto-plasticity. Int. J. Solids Struct. 152, 305-323.

Anand, L., 1983. Plane deformations of ideal granular materials. J. Mech. Phys. Solids 31 (2), 105-122.

Loret, B., 1992. Does deviation from deviatoric associativity lead to the onset of flutter instability? J. Mech. Phys. Solids 40 (6), 1363-1375.

Lade, P.V., Nam, J., Hong, W.P., 2009. Interpretation of strains in torsion shear tests. Comput. Geotech. 36 (1-2), 211-225.

Gutierrez, M., Ishihara, K., Towhata, I., 1991. Flow theory for sand during rotation of principal stress direction. Soils Found. 31 (4), 121-132.

Gutierrez, M., Ishihara, K., 2000. Non-coaxiality and energy dissipation in granular materials. Soils Found. 40 (2), 49-59.

Darve, F., 1990. The expression of rheological laws in incremental form and the main classes of constitutive equations. Geomater. Constitut. Equ. Model. 123148 .

Darve, F., Nicot, F., 2005. On incremental non-linearity in granular media: phenomenological and multi-scale views (part i). Int. J. Numer. Anal. Methods Geomech. 29 (14), 1387-1409.

De Josselin de Jong, G., 1959. Statics and kinematics in the failable zone of a granular material (PhD Thesis). University of Delft, The Netherlands.

Drescher, A., de Josselin de Jong, G., 1972. Photoelastic verification of a mechanical model for the flow of a granular material. J. Mech. Phys. Solids 20 (5), 337-340.

Yoshimine, M., Ishihara, K., Vargas, W., 1998. Effects of principal stress direction and intermediate principal stress on undrained shear behavior of sand. Soils Found. 38, 179-188.

Yang, Z.X., Li, X.S., Yang, J., 2007. Undrained anisotropy and rotational shear in granular soil. Géotechnique 57, 371-384.

Yu, H.S., 2006. Plasticity and Geotechnics. Springer Science \& Business Media.

Spencer, A.J.M., 1964. Theory of the kinematics of ideal soils under plane strain conditions. J. Mech. Phys. Solids 12, 337-351.

Mehrabadi, M.M., Cowin, S.C., 1978. Initial planar deformation of dilatant granular materials. J. Mech. Phys. Solids 26, 269-284.

Harris, D., 1995. A unified formulation for plasticity models of granular and other materials. Proc. Roy. Soc. Lond. Ser. A: Math. Phys. Sci. 450 (1938), 37-49.

Nemat-Nasser, S., Zhang, J., 2002. Constitutive relations for cohesionless frictional granular materials. Int. J. Plast. 18 (4), 531-547.

Zhu, H., Mehrabadi, M.M., Massoudi, M., 2007. The frictional flow of a dense granular material based on the dilatant double shearing model. Comput. Math. Appl. 53 (2), 244-259.

Yu, H., Yuan, X., 2006. On a class of non-coaxial plasticity models for granular soils. Proc. Roy. Soc. A: Math., Phys. Eng. Sci. 462, 725-748.

Tsutsumi, A., Hashiguchi, K., 2005. General non-proportional loading behavior of soils. Int. J. Plast. 21, 1941-1969.

Tsutsumi, S., Kaneko, K., Toyosada, M., Hashiguchi, K., Kishino, Y., 2005. Non-coaxial constitutive response of idealized $3 \mathrm{~d}$ granular assemblies to rotation of principal stress axes. J. Appl. Mech. 8, 565-571.

Li, X., Dafalias, Y., 2004. A constitutive framework for anisotropic sand including non-proportional loading. Geotechnique 54 (1), 41-55.

Dafalias, Y.F., Taiebat, M., 2016. Sanisand-z: zero elastic range sand plasticity model. Géotechnique 66 (12), 999-1013.

de Boer, R., Brauns, W., 1990. Kinematic hardening of granular materials. IngenieurArchiv 60 (7), 463-480.

Papamichos, E., Vardoulakis, I., 1995. Shear band formation in sand according to non-coaxial plasticity model. Geotechnique 45 (4), 649-661.
Qian, J., You, Z., Huang, M., Gu, X., 2013. A micromechanics-based model for estimating localized failure with effects of fabric anisotropy. Comput. Geotech. 50, 90-100.

Zhu, H., Mehrabadi, M.M., Massoudi, M., 2007. A comparative study of the hypoplasticity and the fabric-dependent dilatant double shearing models for granular materials. Int. J. Numer. Anal. Methods Geomech. 31 (5), 735-756.

Li, X., Yu, H.-S., 2015. Particle-scale insight into deformation noncoaxiality of granular materials. Int. J. Geomech. 15 (4), 04014061.

Gao, Z., Zhao, J., 2017. A non-coaxial critical-state model for sand accounting for fabric anisotropy and fabric evolution. Int. J. Solids Struct. 106-107, 200-212.

Shi, J., Guo, P., Stolle, D., 2020. Micromechanical analysis of non-coaxiality between stress and strain increment in granular materials. Acta Geotech., 1-16

Balendran, B., Nemat-Nasser, S., 1993. Double sliding model for cyclic deformation of granular materials, including dilatancy effects. J. Mech. Phys. Solids 41 (3), 573-612.

Li, X., Yu, H.-S., 2014. Fabric, force and strength anisotropies in granular materials: a micromechanical insight. Acta Mech. 225 (8), 2345-2362.

Gudehus, G., 1979. A comparison of some constitutive laws for soils under radially symmetric loading and unloading. Can. Geotech. J. 20, 502-516.

Hareb, H., Doanh, T., 2012. Probing into the strain induced anisotropy of hostun RF loose sand. Granul. Matter 14 (5), 589-605.

Cundall, P.A., Strack, O.D.L., 1979. A discrete numerical model for granular assemblies. Géotechnique 29 (1), 47-65.

Bardet, J.P., 1994. Numerical simulations of the incremental responses of idealized granular materials. Int. J. Plast. 10 (8), 879-908.

Calvetti, F., Viggiani, G., Tamagnini, C., 2003. A numerical investigation of the incremental behavior of granular soils. Rivista Italiana di Geotecnica 37 (3), 11 29.

Froiio, F., Roux, J.N., 2010. Incremental response of a model granular material by stress probing with DEM simulations. In: Goddard, J.D., Jenkins, J.T., Giovine, P. (Eds.), IUTAM-ISIMM Symposium on Mathematical Modeling and Physical Instances of Granular Flow, Sep 2009, Reggio Calabria, Italy, AIP Conference Proceedings, vol. 1227, American Institute of Physics, pp. 183-197..

Wan, R., Pinheiro, M., 2014. On the validity of the flow rule postulate for geomaterials. Int. J. Numer. Anal. Methods Geomech. 38 (8), 863-880.

Darve, F., Nicot, F., 2005. On flow rule in granular media: phenomenological and multi-scale views (part ii). Int. J. Numer. Anal. Methods Geomech. 29 (14), $1411-1432$.

Li, X.S., Dafalias, Y., 2020. Noncoaxiality between two tensors with application to stress rate decomposition and fabric anisotropy variable. J. Eng. Mech. 146 (3), 04020004.

Itskov, M., 2000. On the theory of fourth-order tensors and their applications in computational mechanics. Comput. Methods Appl. Mechods Eng. 189 (2), 419438.

Goddard, J.D., Bashir, Y.M., 1990. On Reynolds dilatancy. In: deKee, D., Kaloni, P.M. (Eds.), Recent Developments in Structured Continua, II. Longman Scientific \& Technical, pp. 23-35.

Bashir, Y.M., Goddard, J.D., 1991. A novel simulation method for the quasi-static mechanics of granular assemblages. J. Rheol. 35, 849-885.

Kruyt, N.P., 1993. Towards micro-mechanical constitutive relations for granular materials. In: Kolymbas, D. (Ed.), Modern Approaches to Plasticity. Elsevier, pp. $147-178$.

Kruyt, N.P., Rothenburg, L., 2004. Kinematic and static assumptions for homogenization in micromechanics of granular materials. Mech. Mater. 36 (12), 1157-1173.

Horne, M.R., 1965. The behaviour of an assembly of rotund, rigid, cohesionless particles. i and ii. Proc. Roy. Soc. Lond. A 286 (1404), 62-97.

Pastor, M., Zienkiewicz, O., Chan, A., 1990. Generalized plasticity and the modelling of soil behaviour. Int. J. Numer. Anal. Methods Geomech. 14 (3), 151-190.

Nicot, F., Darve, F., 2007. Basic features of plastic strains: from micro-mechanics to incrementally nonlinear models. Int. J. Plast. 23 (9), 1555-1588.

Taylor, G.I., 1938. Plastic strain in metals. J. Inst. Metals 62, 307-324.

Bishop, J., Hill, R., 1951. A theoretical derivation of the plastic properties of a polycrystalline face-centred metal. Lond., Edinburgh Dublin Philos. Mag. J. Sci. 42 (334), 1298-1307.

Bishop, J., Hill, R., 1951. A theory of the plastic distortion of a polycrystalline aggregate under combined stresses. Lond., Edinburgh Dublin Philos. Mag. J. Sci. 42 (327), 414-427.

Koiter, W.T., 1953. Stress-strain relations, uniqueness and variational theorems for elastic-plastic materials with a singular yield surface. Quart. Appl. Math. 11 (3), 350-354.

Harthong, B., Wan, R.G., 2013. Directional plastic flow and fabric dependencies in granular materials. In: AIP Conference Proceedings, vol. 1542, AIP, pp. 193-196.

Loret, B., 1990. Geomechanical applications of the theory of multimechanisms. In: Geomaterials-Constitutive Equations and Modelling. Elsevier, New York/NY, pp. $187-211$.

Nemat-Nasser, S., 1983. On finite plastic flow of crystalline solids and geomaterials. J. Appl. Mech. 50 (4b), 1114-1126.

Anandarajah, A., 2008. Multi-mechanism anisotropic model for granular materials. Int. J. Plast. 24 (5), 804-846.

Fu, P., Dafalias, Y.F., 2011. Study of anisotropic shear strength of granular materials using dem simulation. Int. J. Numer. Anal. Methods Geomech. 35 (10), 10981126. 
Šmilauer, V., Catalano, E., Chareyre, B., Dorofeenko, S., Duriez, J., Gladky, A., Kozicki, J., Modenese, C., Scholtès, L., Sibille, L., et al., 2010. Yade reference documentation. Yade Document. 474, 1-531.

Bagi, K., 1996. Stress and strain in granular assemblies. Mech. Mater. 22 (3), 165177.

Weber, J., 1966. Recherches concernant les contraintes intergranulaires dans les milieux pulvérulents. Bul. liaison P. et Ch 2 (64), 170.

Rothenburg, L., Bathurst, R., 1989. Analytical study of induced anisotropy in idealized granular materials. Géotechnique 39 (4), 601-614.

Pouragha, M., Kruyt, N.P., Wan, R., 2019. Fabric response to strain probing in granular materials: Two-dimensional, isotropic systems. Int. J. Solids Struct. 156, 251-262.

Pouragha, M., Wan, R., 2018. On elastic deformations and decomposition of strain in granular media. Int. J. Solids Struct. 138, 97-108.
Dafalias, Y.F., Feigenbaum, H.P., 2011. Biaxial ratchetting with novel variations of kinematic hardening. Int. J. Plast. 27 (4), 479-491.

Yamada, Y., Ishihara, K., 1979. Anisotropic deformation characteristics of sand under three dimensional stress conditions. Soils Found. 19 (2), 79-94.

Ochiai, H., Lade, P.V., 1983. Three-dimensional behavior of sand with anisotropic fabric. J. Geotech. Eng. 109 (10), 1313-1328.

Rycroft, C.H., Kamrin, K., Bazant, M.Z., 2009. Assessing continuum postulates in simulations of granular flow. J. Mech. Phys. Solids 57 (5), 828-839.

Mehrabadi, M., Cowin, S., 1981. On the double-sliding free-rotating model for the deformation of granular materials. J. Mech. Phys. Solids 29 (4), 269-282.

Kruyt, N.P., 1990. An analysis of the generalized double-sliding models for cohesionless granular materials. J. Mech. Phys. Solids 38 (1), 27-35.

Szabó, L., 2016. A note on cross product between two symmetric second-order tensors. J. Mech. Mater. Struct. 12 (2), 147-158. 